\title{
La relación entre el texto original y su traducción: extranjerización de V. Nabokov y domesticación de M. Tsvetaeva
}

\author{
Vladimer LUARSABISHVILI \\ Universidad Estatal de Ilia (Georgia) \\ tato_luarsabishvili@iliauni.edu.ge
}

Recibido: Mayo de 2014

Aceptado: Diciembre de 2014

\begin{abstract}
Resumen
La traducción ha atraído a profesionales, escritores y aficionados desde tiempos remotos. Desde el primer momento se encendieron los debates sobre la técnica de la traducción, desde Cicerón y Horacio, en los tiempos de Bayt al-hikma y San Jerónimo, hasta el siglo XX, los trabajos de L. Venuti, las teorías filosóficas o la traducción audiovisual. En este artículo intentaremos acercarnos a un asunto tan interesante como la relación entre el texto original y su traducción. Como modelos para este estudio tomaremos los ejemplos de las traducciones llevadas a cabo por V. Nabokov y M. Tsvetaeva. Analizaremos la evolución de la doctrina de traducción de V. Nabokov y las causas que influyeron en la decisión de M. Tsvetaeva de domesticar un texto original.masculino.
\end{abstract}

Palabras clave: domesticación, extranjerización, Nabokov, Tsvetaeva, Lorca.

The relation between original text and its translation: foreignization of $V$. Nabokov

and domestication of $M$. Tsvetaeva

\begin{abstract}
The translation was a sphere of interest of professionals, writers and non-professionals during many centuries. From that times the debates about methods of translation originate, including point of views of Cicero, Horace, in time of Bayt al-hikma and St. Jerome, continued in XX century, works of L. Venuti, philosophical theories and audiovisual translation. The goal of our article is to establish the relation between original and translated texts. As an example of the investigation the translations performed by V. Nabokov and M. Tsvetaeva are taken. We analyze the evolution of translational doctrine of V. Nabokov and evaluate the possible reasons which influenced on $\mathrm{M}$. Tsvetaeva to produce the domesticated translation.
\end{abstract}

Key words: domestication, foreignization, Nabokov, Tsvetaeva, Lorca.

SUMARIO: Introducción; 1. Breve introducción a la historia de la traducción; 2. Nabokov sobre Pushkin; 3. Tsvetaeva sobre Lorca; 4. Conclusiones; 5 . Referencias bibliográficas. 


\section{Introducción}

La traducción ha atraído a profesionales, escritores y aficionados desde tiempos remotos. Desde un primer momento se encendieron los debates sobre la técnica de la traducción, desde Cicerón y Horacio, desde los tiempos de Bayt al-hikma y San Jerónimo, hasta del siglo XX, teorías tales como las teorías funcionales (por ejemplo, de Skopos), la aproximación al análisis del registro y discurso (el modelo de Hallidayan, el modelo de House, el análisis del texto y del nivel pragmático de Baker o los trabajos de Hatim y Mason), las teorías sistémicas (la teoría polisistémica, las normas de traducción de Chesterman o la Escuela de la Manipulación), las teorías postcoloniales y de género, los trabajos de L. Venuti, las teorías filosóficas o la traducción audiovisual.

En nuestro artículo intentaremos acercarnos a dos doctrinas de la traducción,denominadas domesticación y extranjerización, basándonos en las obras traducidas por Vladimir Nabokov y Marina Tsvetaeva. Entre las traducciones de V. Nabokov analizaremos el texto de Lewis Carroll Las aventuras de Alicia en el país de las maravillas y de Aleksandr Pushkin Eugenio Oneguin, y en el caso de la traducción de M. Tsvetaeva hemos elegido el poema de Federico García Lorca "Pueblo". Trataremos la primera traducción de V. Nabokov (el texto de L. Carroll) y la traducción de M. Tsvetaeva como ejemplos de domesticación, mientras que la segunda traducción de V. Nabokov será un ejemplo de extranjerización. Como colofón a este artículo, ofreceremos nuestras preferencias por la técnica de extranjerización y la utilizaremos para llevar a cabo una nueva traducción del poema de Lorca "Pueblo".

\section{Breve introducción a la historia de la traducción}

Veamos brevemente la parte de la historia de la traducción que está relacionada con el tema de nuestra investigación.

Amechi Thenacho nos muestra el papel de la interpretación en la comunicación entre diversos grupos lingüísticos que se practicó desde los tiempos de Babel:

Interpretation has been needed and, in fact, practiced since Babel, that is, since two human beings or any two groups of human beings found themselves using languages that were no longer intelligible to each other (IHENACHO 1979: 63).

En el antiguo imperio de Egipto, en la zona de Assuán, se pueden apreciar las inscripciones referidas a intérpretes de aquel tiempo. La convivencia de distintos pueblos en un mismo espacio facilitó la aparición de los intérpretes. La tradición se desarrolló con el paso del tiempo, los intérpretes se hicieron imprescindibles para el comercio, la diplomacia o la guerra.

A distinción de los egipcios, los griegos no respetaban mucho la profesión del intérprete. Prefirieron que los extranjeros aprendieran el griego en lugar de traducir sus propios textos. 
Durante el imperio romano se dio un salto cualitativo importante, los intérpretes fueron especialistas muy respetados. Aparecieron autores tales como Ennio o Cicerón que traducían textos al latín.

La distinción entre las distintas técnicas de traducción comenzó con el texto de Cicerón con el que el mismo autor introdujo sus propias traducciones de los oradores áticos:

Nec converti ut interpres, sed ut orator, sententiis isdem et earum formis tamquam figuris, verbis ad nostram consuetudinem aptis. In quibus non verbum pro verbo necesse habui reddere, sed genus omne verborum vimque servavi ${ }^{1}$.

En su famoso texto Ars Poetica Horacio subrayó la importancia de obtener un texto comprensible como producto de la traducción. Revisando traducciones del Nuevo Testamento del latín y volviendo al hebreo en caso del Antiguo Testamento, San Jerónimo formuló su estrategia en De optimo genere interpretandi:

Ego enim non solum fateor, sed libera voce profiteor, me in interpretatione Graecorum, absque scripturis sanctis, ubi et verborum ordo et misterium est, non verbum e verbo, sed sensum exprimere de sensu².

La labor traductológica realizada por el líder religioso Dao'an es impresionante. En el prefacio de Prajñāpāramitā el autor indica cinco elementos que pueden causar un desvío del texto original y tres factores donde se precisa una especial atención ${ }^{3}$.

Durante el período Abbāsid (750-1250) los traductores cultivaron dos métodos de la traducción - uno, muy literal, por el que traducían palabra por palabra, y en ausencia de un término correspondiente en la lengua meta prestaban la correspondiente palabra griega a la lengua árabe.

Es imprescindible mencionar la escuela de traductores de Toledo del siglo XII, con las épocas raimundiana y alfonsina; durante la primera se tradujeron mayormente textos filosóficos, y en la segunda textos científicos.

García Yebra nos indica la importancia de la labor traductora que se desarrolló en el siglo XVI: "Nunca se han publicado en España, proporcionalmente, tantas traducciones del italiano como en el siglo XVI" ( GARCIA 1983: 324). Este siglo también

\footnotetext{
1 "And I did not translated them as an interpreter, but as an orator, keeping the same ideas and forms, or as one might say, the 'figures' of thought, but in language which conformes to our usage. And in so doing, I did not hold it necessary to render word for word, but I preserved the general style and force of the language" [Cicero, M. T. (46BCE/1960 CE), "De optimo genere oratorum", in Cicero, De inventione, De optimo genere oratorum, topica, translated by H. M. Hubbell, Harvard University Press, Cambridge, MA - Heinemann, London, pp. 347-73; citado en MUNDAY 2008: 19].

2 "Now I not only admit but freely announce that in translation from the Greek - except of course in the case of the Holy Scripture, where even the syntax contains a mystery - I render not word-for-word, but sense-for-sens" [Jerome, E. H. (St Jerome) (395 CE/1997), "De optime genere interpretandi” (Letter 101, to Pammachius), in Epistolae D. Hieronymi Stridoniensis, Aldi F., Rome, 1565, pp. 285-91; translated by P. Carrol as 'on the best kind of translator', in D. Robinson (ed.) (1997b), pp. 22-30; citado en MUNDAY (2008: 20)].

3 Sobre el tema ZÜRCHER, E. (2007): The Buddhist Conquest of China: The spread and adaptation of Buddhism in early medieval China, 3rd edition with a foreword by S. F. Teiser, Brill, Leiden.
} 
es muy importante para los traductores franceses: son prominentes las figuras del traductor Amyot y de autores como Montaigne y Bellay ${ }^{4}$.

En el siglo XVII la traductología avanzó como disciplina en las obras de Denham, Cowley y Dryden. Denham indica que es importante traducir el espíritu de la poesía y no la forma, concediendo al traductor un trato de igual significancia que al autor original y permitiéndole sacar del texto original y reproducir lo que en su opinión sea importante (HERVÁS 1998: 263). Lo mismo señala Cowley, en su ataque a la poesía traducida 'palabra por palabra' (MUNDAY 2008: 25). Dryden definió tres tipos de traducción, siguiendo su terminología, metáfrasis, paráfrasis e imitación, indicando el significado de cada término y la táctica utilizada por el traductor:

All Translation I suppose may be reduced to these three heads. First, that of Metaphrase, or turning an Author word by word, and Line by Line, from one Language into another. Thus, or near this manner, was Horace his Art of Poetry translated by Ben. Johnson. The second way is that of Paraphrase, or Translation with Latitude, where the Authour it kept in view by the Translator, so as never to be lost, but his words are not so strictly follow'd as his sense, and that too is admitted to be amplified, but not alter'd. Such is Mr. Wallers Translation of Virgils Fourth Aeneid. The Third way is that of Imitation, where the Translator (if now he has not lost that Name) assumes the liberty not only to vary from the words and sense, but to forsake them both as he sees occasion: and taking only some general hints from the Original, to run division on the ground-work, as he pleases. Such is Mr. Cowleys practice in turning two odes of Pindar, and one of Horace into English (VENUTI, 2004: 38).

Es bien conocida la doctrina de la traducción de Goethe, que este formuló en su famoso texto:

Hay dos máximas para traducir: la primera pretende que el autor de una nación extranjera sea traspuesto a la nuestra de tal manera que podamos considerarlo como nuestro. La otra, por el contrario, exige de nosotros que nos traslademos a su figura, que nos situemos en sus circunstancias, su manera de decir y sus peculiaridades (VEGA 2004: 266).

La idea fue desarrollada por el teólogo alemán F. Schleiermacher quien estudiaba el carácter mítico de la traducción con otros filósofos y escritores. En 1813 Schleiermacher escribió un artículo interesante que luego se convirtió en texto fundamental para la teoría de la traducción. Schleiermacher destacó dos tipos de traductor y mostró ciertas vías de la traducción:

Pero, entonces, ¿qué caminos puede emprender el verdadero traductor, que quiere aproximar de verdad a estas dos personas tan separadas, su escritor original y su propio lector, y facilitar a este último, sin obligarle a salir del círculo de su lengua materna, el más exacto y completo entendimiento y goce del primero? A mi juicio, sólo hay dos. $\mathrm{O}$

\footnotetext{
4 Sobre el tema CARY, E. (1963): Les grands traducteurs français, Librairie de l'Université, Genève.
} 
bien el traductor deja al escritor lo más tranquilo posible y hace que el lector vaya a su encuentro, o bien deja lo más tranquilo posible al lector y hace que vaya a su encuentro el escritor (VEGA 2004: 251).

El texto de Schleiermacher influyó notablemente en el desarrollo de la teoría. Según H. Kittel y A. Polterman ${ }^{5}$ todas las teorías contemporáneas de la traducción - de origen alemán como mínimo- están basadas en las ideas de Schleiermacher (MUNDAY 2008: 29). Además, en la doctrina de Schleiermacher fundó su teoría de tipología del texto K. Reiss ${ }^{6}$ y también se basó en ella L. Venuti ${ }^{7}$.

Basándose en el concepto de equivalencia y entendiendo el texto como medio de comunicación, Katharina Reiss sistematizó la valoración de los textos y resumió las características básicas de los tipos de textos:

1. 'Plain communication of facts': information, knowledge, opinions, etc. The language dimension used to transmit the information is logical or referential, the content or 'topic' is the main focus of the communication, and the text type is informative.

2. 'Creative composition': the author uses the aesthetic dimension of language. The author or 'sender' is foregrounded, as well as the form of the message, and the text type is expressive.

3. 'Inducing behavioral responses': the aim of the appellative function is to appeal to or persuade the reader or 'receiver' of the text to act in a certain way. The form of language is dialogic, the focus is appellative and Reiss calls this text type operative.

4. Audio medial texts, such as films and visual and spoken advertisements which supplement the other three functions with visual images, music, etc. (MUNDAY 2008: 72).

Hablando del término "invisibilidad" Venuti se refiere a la tradición traductológica en la cultura anglo-americana. Según Munday, "invisibilidad” puede ser producida por dos vías:

by the way translators themselves tend to translate 'fluently' into English, to produce an idiomatic and 'readable' TT, thus creating an 'illusion of transparency';

by the way the translated texts are typically read in the target culture:

A translated text, whether prose or poetry, fiction or non-fiction, is judged acceptable by most publishers, reviewers and readers when it reads fluently, when the absence of any linguistic or stylistic peculiarities makes it seem transparent, giving the appearance that it reflects the foreign writer's personality or intention or the essential meaning of the

\footnotetext{
5 KITTEL H., POLTERMAN, A. (1998): “The German Tradition”, in M. BAKER (ed.) (1998: 418-428).

${ }^{6}$ REISS, K. (1971/2000) Möglichkeiten und Grenzen der Übersetzungskritik, M. Hueber, Munich, translated (2000) by E.F.Rhodes as Translation Criticism: Potential and Limitations, St. Jerome and American Bible Society, Manchester; REISS, K. (1976): Texttyp und Übersetzungsmethode: Der operative Text, Scriptor Verlag, Kronberg; Reiss, K. (1977/89): 'Text types, translation types and translation assessment', translated by A. Chesterman, in A.Chesterman (ed.) (1989:105-115); REISS, K. (1981/2004): 'Type, kind and individuality of text: desicion making in translation', translated by S.Kitron, in L.VENUTI(ed.) 2004: 168-179.

7 VENUTI, L. (ed.) (1992): Rethinking translation: Discourse, Subjectivity, Ideology, Routledge, London \& New York; VENUTI, L. (1995/2008): The Translator's Invisibility: A History of Translation, Routledge, London \& New York.
} 
foreign text - the appearance, in other words, that the translation is not in fact a translation, but the 'original' (MUNDAY 2008: 144).

Venuti afirma que son las editoriales quienes determinan las técnicas y la labor de los traductores, puesto que son ellas las que aceptan o rechazan la traducción. El resultado es que las características de la cultura original suelen diluirse en el texto traducido y el producto que recibimos está adaptado al máximo a la cultura meta. A esto Venuti lo denomina la domesticación 8 y lo caracteriza como "an ethnocentric reduction of the foreign text to (Anglo-American) target-language cultural values" (VENUTI 1995: 20). Además, a través de la domesticación, el lector reconoce su propia cultura en el texto traducido, su propia cultura en otras culturas. Para Venuti este tipo de traducción no es más que una falsificación del texto original, que se distingue de la otra técnica, la de extranjerización.

Por medio de la extranjerización el texto traducido conserva las características de la cultura original y, además, detiene la relación etnocéntrica en la traducción. En palabras de Venuti: "they are equally partial (as are domesticating translations) in their interpretation of the foreign text, but they do tend to flaunt their partiality instead of concealing it" (VENUTI 1995: 34).

\section{Nabokov sobre Pushkin}

En un artículo "Nabokov as translator", L. Kimmel examina la evolución de la doctrina de traducción de Vladimir Nabokov. La primera parte del artículo está dedicada a la investigación del texto de L. Carroll Las aventuras de Alicia en el país de las maravillas ${ }^{9}$. En el original, Carroll con frecuencia juega con las palabras basándose en la polisemia y coincidencias fonéticas que componen un efecto humorístico. Durante la traducción Nabokov adaptó el texto original a la cultura meta, empeño que empezó con el cambio de nombre de la protagonista (Ania en lugar de Alice) y continuó con detalles pequeños; con todo, el texto final podría ser de difícil comprensión para el lector ruso. Todo lo mencionado dio lugar a que el texto traducido pueda ser tratado, no como un texto traducido del inglés al ruso, sino como parte de la literatura rusa.

En la segunda parte del artículo L. Kimmel examina otra traducción de V. Nabokov: el Eugenio Oneguin de A. Pushkin. A distinción de la traducción del texto de L. Carroll, esta vez Nabokov decidió guardar la integridad del texto original y tradujo lo más cercano posible al texto, y además añadió al texto traducido comentarios que facilitan la comprensión del texto original a un lector no ruso. Así la traducción

\footnotetext{
8 Según Venuti, la domesticación es habitual en la tradición anglo-americana, mientras que PYM (1996: 170) también la encuentra en otros países -Brasil, España y Francia-, y HATIM (1999: 204) la ve como instrumento habitual en la cultura de los países occidentales.

9 Sobre este tema AMBROSIANI, Per, “«Оставшаяся при отступлении Наполеона»: Domestication, Foreignization, and the Foreign in Russian Translations of Alice's Adventures in Wonderland", talk presented at the conference Domestication and Foreignization in Translation Studies, University of Eastern Finland, Joensuu campus, 29th September 2011 - 1st October 2011; YIFANG, L. (2014): “Análisis de varias traducciones coetáneas de Alice's Adventures in Wonderland a las lenguas china y española”, Sendebar, 24, pp. 169-194.
} 
del texto de A. Pushkin se convirtió para V. Nabokov no en una tarea de traductor sino en un intento de guardar los mensajes culturales y semánticos codificados en el original de la traducción. En el libro The Translation Studies Reader, editado por L. Venuti, está incluido un texto de V. Nabokov sobre los problemas de traducción de Eugenio Oneguin (VENUTI 2004: 115-128) donde Nabokov dice lo siguiente sobre los textos de A. Pushkin:

Pushkin's line is, by-the-by, an excellent illustration of what I mean by "literalism, literality, literal interpretation". I take literalism to mean "absolute accuracy". If such accuracy sometimes results in the strange allegoric scene suggested by the phrase "the letter has killed the spirit", only one reason can be imagined: there must have been something wrong either with the original letter or with the original spirit, and this is not really a translator's concern.

Con respecto a las traducciones al francés, alemán e inglés de Eugenio Oneguin Nabokov escribe lo siguiente:

So here are three conclusions I have arrived at: 1. It is impossible to translate Onegin in rhyme. 2. It is possible to describe in a series of footnotes the modulations and rhymes of the text as well as all its associations and other special features. 3. It is possible to translate Onegin with reasonable accuracy by substituting for the fourteen rhymed tetrameter lines of each stanza fourteen unrhymed lines of varying length, from iambic dimeter to iambic pentameter (VENUTI 2004: 127).

Según L. Kimmel, el cambio de la doctrina de traducción no se dio como resultado del hecho de que la obra de Carroll esté destinada al lector infantil, a diferencia del libro de A. Pushkin, sino por causa de la evolución de la doctrina del traductor. Es bien sabido que V. Nabokov, al traducir sus propios textos, cambiaba el contenido y los títulos (como en caso de Camera obscura, que renombró Laughter in the Dark, y cuyo contenido reescribió durante el proceso de traducción). Según L. Kimmel, es posible que Nabokov llegara a la conclusión de que sólo el autor posee el derecho de modificar un texto original. Sobre una obra maestra Nabokov afirmó lo siguiente:

The person who desires to turn a literary masterpiece into another language, has only one duty to perform, and this is to reproduce with absolute exactitude the whole text, and nothing but the text (VENUTI 2004: 121).

\section{Tsvetaeva sobre Lorca}

Presentamos aquí el original del poema "Pueblo" de Federico García Lorca y su traducción realizada por M. Tsvetaeva (LORCA 2000: 83; LORCA 2010: 57). 


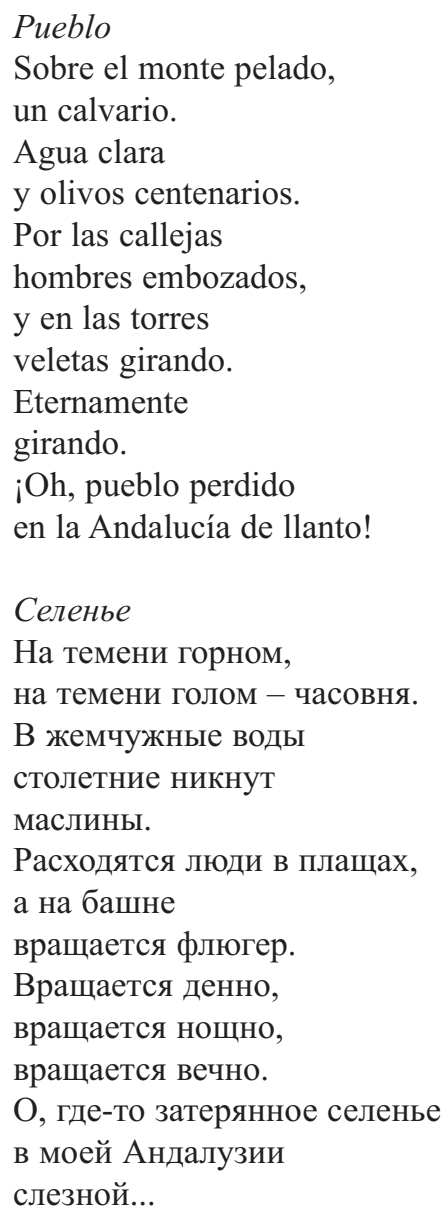

Antoine Berman, autor de un famoso texto ${ }^{10}$, habla sobre la importancia del "receiving foreign as foreign" (que nos hace recordar la extranjerización de Venuti) y menciona "system of textual deformation" en el texto traducido a lo que llama "negative analytic":

The negative analytic is primarily concerned with ethnocentric, annexionist translations and hypertextual translations (pastische, imitation, adaptation, free writing), where the play of deforming forces is freely exercised (BERMAN 2004: 278; MUNDAY 2008: 147).

Teniendo en cuenta la variedad lingüística y creatividad que ocurre durante la traducción, Berman identifica doce "tendencias deformantes" que pueden ser aplicadas en el caso de traducción del poema de Lorca. En particular, la segunda tendencia definida por Berman es clarificación (clarification), que "aims to render 'clear' what

10 L'Épreuve de l'étranger: Culture et traduction dans l'Allemagne romantique (1984). 
does not wish to be clear in the original" (BERMAN 2004: 278; MUNDAY 2008: 281). Tsvetaeva traduce: "Расходятся люди в плащах"; el verbo "Расходятся" nо existe en el texto original, tampoco "в плащах". En el original leemos "embozados" que no necesariamente significa "в плащах"; aquí Tsvetaeva intenta hacer el texto original más claro y ayudar al lector a entender la intención de Lorca, que no está detallada en el texto original.

La tercera tendencia es expansión (expansion), que hace un texto traducido más largo que el original. Tsvetaeva inserta una estrofa entera que no existe en el original: "Вращается денно, вращается нощно, вращается вечно". Haciendo esto, recibimos la domesticación del texto original, la inducción de la rima y la iniciación del núcleo semántico.

La cuarta tendencia es ennoblecimiento (ennoblement) o una tentativa de escribir más elegante, que también utiliza Tsvetaeva: la tercera estrofa empieza "B жемчужные воды столетние никнут маслины". En el texto original no existe "никнут" pero sí una descripción: "Agua clara y olivos centenarios" que es la continuación del núcleo semántico que inicia en la primera estrofa: "Sobre el monte pelado, un calvario"; en la misma estrofa también leemos "жемчужные воды“ que no necesariamente corresponde a "Agua clara".

La séptima tendencia es la destrucción de las rimas (the destruction of rhythms), cuando en el texto traducido la rima original se pierde y es sustituida por una nueva rima. La utilización de la forma "темени" de un modo muy sutil y acertado provoca la inducción de la rima que en el texto original se basa en la simple repetición de las sílabas finales: calv-ario - centen-arios.

Los ejemplos citados muestran la intención de la traductora de llevar a cabo la domesticación del texto original. Pero ¿con qué propósito?

Una respuesta posible podemos encontrarla en un libro de André Lefevere ${ }^{11}$. El autor habla sobre las causas concretas que inducen a la aceptación o al rechazo del texto traducido. Y menciona factores como el poder, la ideología y la manipulación. Por tanto, un texto traducido puede ser reescrito ideológicamente o poéticamente, lo que depende de la ideología o poética dominante del momento en que se realiza la traducción. Un componente ideológico puede estar conectado con otro económico, mientras que la poética dominante puede influir en la recepción y en el rechazo del texto traducido. Es evidente que la estructura de los idiomas ruso y español es diferente y este hecho puede jugar un cierto rol durante el proceso de traducción. Aparte de esta característica, teniendo en cuenta que el poema de Lorca no es muy rimado, pensamos que M. Tsvetaeva pudo haberlo traducido sin elementos de expansión, ennoblecimiento y clarificación. Sin embargo, si tenemos en cuenta las peculiaridades de la industria de la traducción en tiempos de Tsvetaeva, podemos entender el deseo de la famosa poetisa y traductora, de realizar una traducción aceptable en el sentido artístico. Según Venuti, la domesticación induce "an ethnocentric reduction of the foreign text to target-language cultural values" y el "target-language" (VENUTI 1995: 20) en nuestro caso es una poética dominante rusa. Por supuesto, cuando M. Tsvetaeva realizó su traducción la sociedad rusa no estaba dispuesta a recibir la

11 Translation, rewriting and the Manipulation of Literary Fame (1992). 
poesía modernista de Lorca, a pesar de que Rusia fue uno de los países pioneros en la poesía modernista. Al mismo tiempo, debemos tener en cuenta que las traducciones fueron las únicas vías de ingresos para ganarse la vida que tenía Tsvetaeva, porque sus libros eran sistemáticamente censurados. La única posibilidad de sobrevivir para la poetisa fue hacer traducciones, domesticadas y deformadas, pero aceptables. Al mismo tiempo, no hay que olvidar que Tsvetaeva no dominaba el español y traducía utilizando los llamados "podstrochniki" lo que hay que tener en cuenta estimando las traducciones de la poetisa rusa.

Teniendo en cuenta todo lo mencionado, decidimos hacer un experimento de traducción y traducir el poema de Lorca sin domesticarlo. El resultado lo ofrecemos aquí:

\author{
Селенье \\ На голой горе \\ распятие. \\ Прозрачная вода \\ и столетние маслины. \\ По улочкам \\ закутанные люди \\ и на башнях \\ флюгеры вертятся. \\ Вращаются вечно. \\ O, потерянное селенье \\ в Андалусии слезной!
}

La distinción básica entre la traducción de Tsvetaeva y la nuestra es la siguiente: hemos realizado la transferencia cultural traduciendo "por las callejas" como "По улочкам" у "hombres embozados" сото "закутанные люди" (у по "Расходятся люди в плащах" como ofrece la traducción de Tsvetaeva). De este modo, hemos evitado la segunda tendencia citada por Berman -clarificación (clarification)- y hemos permitido a la cultura original pasar a la cultura meta.

También, hemos eludido la cuarta tendencia - ennoblecimiento (ennoblement)-: "Прозрачная вода" corresponde más a "Agua clara" y, al mismo tiempo, no contiene la palabra "никнут", у continúa en "Прозрачная вода и столетние оливы", como en el texto original.

La séptima tendencia -la destrucción de las rimas (the destruction of rhythms)la hemos evitado traduciendo la palabra original "calvario" como "распятие" y $\sin$ incorporar en la primera línea un sustantivo "часовня".

\title{
4. Conclusiones
}

Para finalizar nuestro artículo y para hacer conclusiones necesarias nos parece imprescindible entender las causas concretas que provocan la domesticación del poema de Lorca. Lo primero que debemos tener en cuenta es el período histórico en 
que Tsvetaeva realiza la traducción. En la Rusia de Tsvetaeva el único movimiento cultural (dominante) era el realismo socialista, que no podría aceptar textos vanguardistas. Por otro lado, traducir a un poeta como Lorca era importante, ya que Lorca fue una de las víctimas más dramáticas del régimen franquista.

Basándose en las ideas de los formalistas rusos y el estructuralismo de la escuela de Praga, el profesor israelí Itamar Even-Zohar propuso la teoría del polisistema ${ }^{12}$. En su opinión, las obras literarias forman parte de un sistema literario. Con respecto a la literatura traducida, dice que opera como un sistema y argumenta sus palabras así:

in the way the TL selects works for translation,

in the way translation norms, behavior and policies are influenced by other cosystems (MUNDAY 2001: 108).

La literatura traducida puede ocupar un lugar primario o secundario en el sistema literario. La posición secundaria es una posición periférica y la literatura traducida en la Unión Soviética no podría ocupar otro lugar a pesar de las magníficas traducciones de El Quijote o la poesía georgiana. Decía Even-Zohar que la posición secundaria es la posición "normal" pero destacaba al mismo tiempo que ciertas traducciones pueden llegar a ocupar una posición primaria. Y existen tres posibilidades para que la literatura traducida se asiente en posición primaria:

when a 'young' literature is being established and looks initially to 'older' literatures for ready-made models;

when a literature is 'peripheral' or 'week' and imports those literary types which it is lacking. This can happen when a smaller nation is dominated by the culture of a larger one;

when there is a critical turning point in literary history at which established models are no longer considered sufficient, or when there is a vacuum in the literature of the country. Where no type holds sway, it is easier for foreign models to assume primacy (MUNDAY 2001: 109).

En nuestro caso, la domesticación del texto original de Lorca dio lugar a la pérdida de los elementos de la cultura original y a la composición de una semántica nueva. Lo que resulta es un giro desde la traducción como texto hasta la traducción como cultura, lo que Mary Snell-Hornby denominó "un giro cultural". Es bien sabido que luego el término fue acogido por André Lefevere quien hablaba sobre la reescritura de los textos durante la traducción. Lefevere citaba un ejemplo de traducciones de Omar Jayam realizado por Edward Fitzgerald. Según Lefevere, en un sistema literario las funciones de la traducción son controladas por tres factores mayores: 1) los profesionales del sistema literario; 2) el patrocinio exterior del sistema literario; 3) la poética dominante (MUNDAY 2008: 126). Lo que nos parece crucial en el caso

12 EVEN-ZOHAR, I. (1978/2004): "The position of translated literature within the literary polysystem", in L. VENUTI (ed.) (2004:199-204). 
de la traducción domesticada de Tsvetaeva es el tercer componente -la poética dominante- que, según Munday, Lefevere analiza en dos componentes:

Literary devices: These include the range of genres, symbols, leitmotifs and prototypical situations and characters.

The concept of the role of literature: This is the relations of literature to the social system in which it exists. The struggle between different literary forms is a feature of polysystem theory. Lefevere takes this idea further and looks at the role of institutions in determining the poetics. Lefevere takes this idea further and looks at the role of institutions in determining the poetics:

Institutions enforce or, at least, try to enforce the dominant poetics of a period by using it as the yardstick against which current production is measured. Accordingly, certain works of literature will be elevated to the level of 'classics' within a relatively short time after publication, while others are rejected, some to reach the exalted position of a classic later, when the dominant poetics has changed (MUNDAY 2008: 127).

Durante sus últimos años Tsvetaeva vivía en un exilio interior dedicándose prácticamente sólo a las traducciones. Todos sus libros eran censurados y prohibida su publicación. Debido a eso, traducir fue la única posibilidad que le restaba para ganarse la vida. Sus traducciones tampoco se consideraban del primer nivel, pero tras la abolición del régimen comunista tanto libros como traducciones de la poetisa se convirtieron en textos de éxito. Siguiendo las ideas de Lefevere, hoy en día todos los textos de Tsvetaeva reciben el tratamiento de textos clásicos, después de la evolución y transformación de la poética dominante -el realismo socialista. Aún más, Tsvetaeva tuvo y tiene muchos seguidores en el terreno de la traducción, que continúan traduciendo la poesía española utilizando la técnica de la domesticación. Pero esto ya es tema de otro estudio.

Basándonos en todo lo mencionado, nos parece más viable el empleo de la técnica de extranjerización en el caso de la traducción de los textos poéticos para conseguir una traducción más adecuada.

\section{Referencias bibliográficas}

BAKER, M., MALMKJAER, K. (1998): Encyclopedia of Translation Studies, 1st edition, Routledge, London and New York.

BERMAN, A. (2004): "Translation and the trials of the foreign", in L. Venuti (ed.), The Translation Studies Reader, Routledge, London and New York.

GARCÍA YEBRA, V. (1983): En torno a la traducción: teoría, crítica, historia, Gredos, Madrid.

HATIM, B. (1999): "Implications of Research into Translator Invisibility", Target, II (2), pp. 201-222.

HERVÁS JÁVEGA, I. (1998): “La reflexión traductológica: una revisión diacrónica (I)”, Philologia Hispalensis, 12, pp. 225-267. 
IHENACHO, A. (1979): "The Role of Translation and Interpretation in West Africa”, Babel, 25 (2), pp. 59-71.

KIMMEL, L. (1998): "Nabokov as Translator. An examination of his changing doctrine of translation", [en línea]. www.leighkimmel.com/writing/academicpapers/nabokov2.shtml [Consulta: 31 marzo 2012]

LORCA, F. G. (2010): Poema del cante jondo. Romancero gitano (conferencias y poemas), Stockcero, Doral, FL.

LORCA, F. G. (2000): Poezia, proza, teatr, Institut Di-Dik, Moskva.

MUNDAY, J. (2001): Introducing Translation Studies. Theories and applications, Routledge, London \& New York.

MUNDAY, J. (2008): Introducing Translation Studies. Theories and applications. The Translator's Invisibility, Routledge, London \& New York.

NABOKOV, V. (2004): "Problems of Translation: Onegin in English", en The Translation Studies Reader, Lawrence Venuti (eds.), Routledge, London \& New York, pp. 115-128.

PYM, A. (1996): "Review article: Venuti's Visibility", Target, 8 (I), pp. 165-177.

VEGA, M. A. (2004): Textos clásicos de teoría de la traducción, Cátedra, Madrid. VENUTI, L. (1995): The Translator's Invisibility, Routledge, London \& New York. VENUTI, L. (2004): The Translation Studies Reader, Routledge, London \& New York. 